Decano Facultad de Ciencias Médicas. UNC

Prof. Dr. Marcelo A Yorio

Vice Decana Facultad de Cs. Médicas. UNC

Prof. Dra. Marta Fiol de Cuneo.

Secretaría de Salud Pública y Ambiente

Prof. Dr Gustavo Irico

Directora de la Revista

Prof. Dra. Ma. Cristina Cometto

\section{Comité editorial}

Prof. Mg. María Borsotti

Escuela de Salud Pública. FCM UNC

Prof. Mg. Oscar Mareca

Esc. de Salud Pública. FCM UNC

Prof. Dra. Patricia F. Gómez

Escuela de Enfermería FCM UNC.

Prof. Med. Gustavo Martínez

Municipalidad de Córdoba

Prof. Mg. Rubén Castro Toschi

Esc. de Salud Pública. FCM UNC

Prof. Mg. Abelardo Rahal

Escuela de Salud Pública. FCM UNC

\section{Comité de Redacción}

Prof. Dr. Leandro Dionisio

Esc. de Salud Pública. FCM UNC

Med. Mariela Roldan

Hospital Nacional de Clínicas

Mg. Susana Rivolta

Esc. de Salud Pública FCM UNC

Dr. Ariel Abeldaño

Esc. de Salud Pública FCM UNC

Dra. Monica Bella

Esc. de Salud Pública FCM UNC

Mg. Hugo L. Pizzi

Esc. de Salud Pública FCM UNC

\section{Consejo Científico Nacional}

Prof. Dra. Noemí Bordoni. Directora del Instituto de Investigaciones en Salud Pública. Coordinadora Ejecutiva de la Maestría en Salud Pública. UBA

Prof. Dra. Norma Cristina Meichtry. Doctor of Philosophy (Sociología- Demografía). Profesora Titular Ordinaria en Geografía Argentina UNNE. Directora Instituto de Revista de Salud Pública, (XIX) 1:1, abr

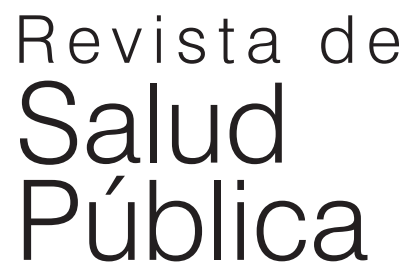

2015 Investigaciones Geohistóricas. Investigadora Independiente en el Inst. de Investigaciones Geohistóricas. CONICET

Dr. Jorge Kiguen Prof. Titular Ordinario de Epidemiologia. Universidad Nacional del Litoral. Rep. A.

Prof. Mg Ana M Heredia. Profesor Titular Regular Escuela de Enfermería. Departamento de Ciencias Sociales. Universidad Nacional de Quilmes

Dra. Silvina Malvarez. Ex consultora de la OPS OMS WDC

Dr. Luis Simes. Instituto Universitario de Ciencias de la salud. Universidad Barceló. Bs. As.

Dr. Bruno Sebastián Ribotta. Investigador Asistente del CONICET. Lugar de trabajo Centro de Investigaciones y Estudio sobre Cultura y Sociedad. CIECS, CONICET y UNC. Universidad Nacional de Córdoba.

\section{Consejo Científico Internacional}

Prof. Dr. Giorgio Solimano. Universidad de Chile

Dr. Charles Godue. Ex consultor OPS. WDC

Prof. Dr. Juan Jesús Gestal Otero. Universidad de Sgo. de Compostela. España

Prof. Dr. José Ramón Martínez Riera. Profesor Titular del Depto. de Enfermería Comunitaria. Medicina Preventiva y Salud Pública e Historia de la Ciencia de la Universidad de Alicante. España. 
La Revista de Salud Pública es una publicación temática, indizada, con arbitraje externo de pares nacionales e internacionales, trimestral, destinada a la publicación de la producción técnico científica relacionada al área de la salud y en especial de la Salud Pública. Editada por la Escuela de Salud Pública de la Facultad de Ciencias Médicas de la Universidad Nacional de Córdoba.

Consta de ISSN 1853-1180 para la versión Impresa y ISSN 1852-9429 para la Electrónica.

The Journal of Public Health is a thematic publication, indexed, external peer refereed national and international, biannual, for the publication of the technical-scientific production related to the area of health and in particular Public Health. Published by the School of Public Health, Faculty of Medical Sciences of the University National Córdoba, ISSN 1853- 1180 consists of the printedversion and for Electronic ISSN 1852-9429.

Bibliotecóloga: Mónica S. Perfetti

Traducción al inglés: Andrea Ternengo

Traducción al portugués: Osvaldo J. Casero

Tesorería y Finanzas: Cdora. Soledad Marquez

Diseño y Diagramación: Vladimir López Barrios

Escuela de Salud Pública. Calle Enrique Barros esq. Enf. Gordillo Gomez CP 5000. Córdoba. República Argentina. Te: 0351 - 4334042 / 4333023

www.saludpublica.fem.unc.edu.ar saludpublica@fcm.unc.edu.ar publicacionesp@fcm.unc.edu.ar ISSN 1853-1180

Las responsabilidades por los juicios, opiniones, puntos de vista, o traducciones; expresados en los artículos publicados corresponden exclusivamente a los autores.

No está permitida la reproducción parcial o total del contenido de la revista, sin la previa autorización de los editores de la Revista de Salud Pública.

Impreso en:

Taller General de Imprenta, UNC Agosto, 2017

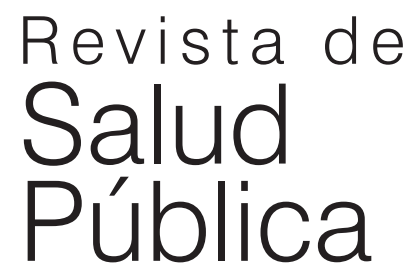

(n)




\section{Sumario}

EDITORIAL

\section{ARTICULOS CIENTIFICOS}

Programas de Salud y Prácticas de cuidado nutricional infantil.

Scruzzi Graciela Fabiana , Lucchese Marcela.

Adaptación transcultural de mensajes vía teléfonos móviles como apoyo para dejar de fumar. María Cristina Ortíz León, James Thrasher, Jaime Morales Romero, Xóchitl De San Jorge Cárdenas.

Estudio bibliométrico de la producción científica argentina en cáncer a través de las bases de datos medline y lilacs.

Rolando Pablo Juárez, Rodolfo Martín Barrere.

Validación del Cuestionario Nórdico Estandarizado de Síntomas Musculoesqueléticos para la población trabajadora chilena, adicionando una escala de dolor. Marta Martínez Maldonado, Rubén Alvarado Muñoz.

Disminución de la fecundidad y cambios en la necesidad insatisfecha de planificación familiar en los condados de América Latina y el Caribe.

Javiera Fanta Garrido.

Factores influyentes en la satisfacción usuaria de la atención primaria en salud latinoamericana: revisión integradora.

Katiuska Reynaldos Grandón, Javiera Achondo Oisel, Valentina Azolas Valenzuela.

Consumo excesivo episódico de alcohol: cambio natural en estudiantes universitarios. Karina Conde, Aldana Lichtenberger, Raquel Inés Peltzer, Mariana Cremonte.

Satisfaccion del usuario en la emergencia del hospital central de maracay.

Gómez G.Wuilman E., Dávila L. Fanny J., Campins R., Rafael A., Colmenarez D., Stefanny.

Tratamiento con inmunoglobulina $g$ intravenosa (iggiv).inmunomodulacion en fallas reproductivas.

Daniela Fontana, Ester Feldman, Aldana Torno, Catalina Massa, Carolina Barros, Roxana Rivero, Silvia Joekes, Alicia Mazzolli, Graciela Kortebani.

UNA MIRADA HISTORICA

Alcoholismo en las tesis de la Facultad de Ciencias Médicas de Córdoba a fines del siglo XIX. Julieta Lucero.

FORO ABIERTO DE OPINION

Conceptos "puente" para el trabajo multidisciplinario en salud pública. María A. Sánchez Bandala.

RESEÑA BIBLIOGRAFICA

Las grandes tendencias demográficas y el ambiente: El desafío de un planeta viable. Roberto Ariel Abeldaño. 


\section{Summary}

EDITORIAL

\section{SCIENTIFIC ARTICLES}

Health Programs and Child Nutritional Care Practices.

Scruzzi Graciela Fabiana, Lucchese Marcela.

Cross-cultural adaptation of text messages as a support tool to quit smoking.

María Cristina Ortíz León, James Thrasher, Jaime Morales Romero, Xóchitl De San Jorge Cárdenas.

Bibliometric Study through Medline and Lilacs Databases of Scientific Argentinian Production on Cancer.

Rolando Pablo Juárez, Rodolfo Martín Barrere.

Validation of the Nordic Standardized Questionnaire of Musculoskeletal Symptoms for the Chilean working population, including a pain scale.

Marta Martínez Maldonado, Rubén Alvarado Muñoz.

Fertility decline and changes in unmet need for family planning in counties of Latin America and the Caribbean.

Javiera Fanta Garrido.

Factors that influence the user satisfaction of Primary Health Care Latin American: Integrative review.

Katiuska Reynaldos Grandón, Javiera Achondo Oisel, Valentina Azolas Valenzuela.

Binge drinking: natural change in university students.

Karina Conde, Aldana Lichtenberger, Raquel Inés Peltzer, Mariana Cremonte.

Satisfaction of users of the emergency room at maracay's central hospital.

Gómez G.Wuilman E., Dávila L. Fanny J., Campins R., Rafael A., Colmenarez D., Stefanny.

Treatment with intravenous immunoglobulin g (iggiv). immunomodulation reproductive failures. Daniela Fontana, Ester Feldman, Aldana Torno, Catalina Massa, Carolina Barros, Roxana Rivero, Silvia Joekes, Alicia Mazzolli, Graciela Kortebani.

\section{HISTORICAL OUTLOOK}

Alcoholism in Theses from the School of Medicine of Cordoba at the End of the 19th Century. Julieta Lucero.

\section{OPEN FORUM OF OPINION}

"Bridge" concepts for multidisciplinary work in public healh. María A. Sánchez Bandala.

\section{BIBLIOGRAPHICAL REVIEW}

"The great demographic tendencies and the environment: The challenge of a viable planet. Roberto Ariel Abeldaño.

\section{RULES FOR THE PRESENTATION OF PAPERS}


Programas de saúde e práticas de cuidados de nutrição infantil.

Scruzzi Graciela Fabiana , Lucchese Marcela.

Adaptação transcultural de mensagens via celular para apoiar a cessação do tabagismo. María Cristina Ortíz León, James Thrasher, Jaime Morales Romero, Xóchitl De San Jorge Cárdenas.

Estudo bibliométrico da produção científica argentina em câncer através dos bancos de dados medline e lilacs.

Rolando Pablo Juárez, Rodolfo Martín Barrere.

Validação do questionário padronizado de síndrome musculoesquelético nórdico para a população trabalhadora chilena, adicionando uma escala de dor.

Marta Martínez Maldonado, Rubén Alvarado Muñoz.

Declínio da fertilidade e mudanças nas necessidades insatisfeitas de planejamento familiar nos municípios da América Latina e Caribe.

Javiera Fanta Garrido.

Fatores influentes na satisfação do usuário da Atenção Primária na Saúde da América Latina: revisão integrativa.

Katiuska Reynaldos Grandón, Javiera Achondo Oisel, Valentina Azolas Valenzuela.

Consumo excessivo episódico de álcool: mudança natural em estudantes universitários. Karina Conde, Aldana Lichtenberger, Raquel Inés Peltzer, Mariana Cremonte.

Satisfação de o usuário na emergência do hospital central de maracay.

Gómez G.Wuilman E., Dávila L. Fanny J., Campins R., Rafael A., Colmenarez D., Stefanny.

Tratamento com inmunoglobulina $\mathrm{g}$ im intrutiva (iggiv) .munomodulação em falhas reprodutivas.

Daniela Fontana, Ester Feldman, Aldana Torno, Catalina Massa, Carolina Barros, Roxana Rivero, Silvia Joekes, Alicia Mazzolli, Graciela Kortebani.

UM OLHAR HISTÓRICO

Alcoolismo nas teses da Faculdade de Ciências Médicas de Córdoba no final do século. Julieta Lucero.

FORUM ABERTO OPINIÃO

Conceitos «ponte» para o trabalho multidisciplinar na saúde pública. María A. Sánchez Bandala.

\section{REVISÃO BIBLIOGRÁFICA}

As grandes tendências demográficas e o ambiente: o desafio de um planeta viável.. Roberto Ariel Abeldaño. 


\section{Revista indizada en base de datos}

\section{LILACS}

Literatura Latinoamericana y del Caribe en Ciencias de la Salud. Bireme, Sistema

Latinoamericano y del Caribe de Información en Ciencias de la Salud, Brasil. http://www.bireme.br/

\section{CUIDEN}

Base de Datos Bibliográfica sobre Cuidados de Salud en Iberoamérica. Base de datos de enfermería en español, Granada - España http://www.doc6.es/index/

\section{PERIODICA}

Base de datos de la Dirección General de Bibliotecas de la UNAM. Universidad Nacional Autónoma de México. http://132.248.9.1:8991/F/-/?func=find-b0\&local_base=PER01

\section{RENICS}

Red Nacional de Información en Ciencias de la Salud. http://www.renics.com.ar/

DOAJ

Directory of open access journals Universidad Lund, Suecia http://www.doaj.org/

NÚCLEO BÁSICO DE REVISTAS CIENTÍFICAS ARGENTINAS. CAYCITCONICET. R. Argentina HTTP://www.caicyt-conicet.gov.ar/nucleo-basico-derevistas-cientificas/

SISTEMA NACIONAL DE REPOSITORIOS DIGITALES - Biblioteca Electrónica de Ciencia y Tecnología del Ministerio de Ciencia, Tecnología e Innovación Productiva (Mincyt). Se accede a través de este link:

http://www.biblioteca.mincyt.gob.ar/revistas/index?letter $=\&$ title $=$ revista + de $+\mathrm{s}$ alud + publica\&openaccess $=$ false $\&$ gran_area $=3 \&$ area $=\& v i e w=l i s t \& y t 0=$ Busca

Revistas de la Universidad Nacional de Córdoba espacio destinado a revistas electrónicas, de acceso librema la produccion cientifica, un recurso publico, es por ello que somos parte de este sitio que la universidad ofrece de acceso libre a la produc

El sistema con que opera este sitio es Open Journal Systems (OJS).

Se accede: http://revistas.unc.edu.ar/ 